\title{
SUGGESTIONS CONCERNANT LE RÉAMÉNAGEMENT DES CARRIËRES EN EAU
}

\author{
J.-L. MERIAUX (1), P. TOMBAL (2)
}

\section{RÉSUMÉ}

L'intégration harmonieuse des carrières en eau dans les paysages fluviatiles, leur réaménagement à des fins halieutiques ou touristiques, voire conservatoires, nécessite la prise en compte de l'état présent de leur environnement, mais surtout de ses potentialités. Les suggestions concernent:

- la taille et la forme des plans d'eau à modeler après extraction des graves et maintien en eau ;

- la définition des profils des berges permettant un développement rapide de la végétation et la réinstallation d'une faune diversifiée:

- le choix des essences végétales à introduire, en particulier dans les vallées de la Somme, de l'Oise et de l'Aisne et les techniques à employer, exprimés sur la base de l'étude d'une vingtaine de cas situés dans les vallées de la Somme et de l'Oise.

\section{INTRODUCTION}

Lors d'une demande d'ouverture ou d'extension de carrière, il est nécessaire que le pétitionnaire ou maître d'ouvrage joigne à la procédure normale remise à l'Administration une étude d'impact, ceci conformément à la loi du 10 juillet 1976 relative à la Protection de la Nature (décret d'application du 12 octobre 1977) et en regard du Code minier notamment à son article 106 modifié par la loi du 16 juin 1977 (décret d'application du 20 décembre 1977). Le point 4 de 1'article 2 détaillant le contenu de l'étude d'impact stipule que cette étude doit présenter "les mesures envisagées par le maître d'ouvrage ou le pétitionnaire pour supprimer, réduire et si possible compenser les conséquences dommageables du projet sur l'environnement, ainsi que l'estimation des dépenses correspondantes ". Le réaménagement du site après exploitation est donc une condition nécessaire et indispensable à l'obtentioń de la demande d'ouverture ou d'extension de la carrière. Si cette. réintégration du site dans le paysage est toujours abordée (théoriquement plus ou moins bien d'ailleurs), d'un point de vue pratique, sa réalisation est exceptionnellement conçue de façon logique, en particulier pour les carrières installées dans les grandes vallées et qui demeurent en eau après extraction des graves. Nous avons donc essayé dans cette note de présenter un ensemble de suggestions qui peuvent faciliter ou permettre un réaménagement rationnel des carrières en eau, notamment à des fins halieutiques ou touristiques mais aussi conservatoires, et leur intégration dans les grands paysages fluviatiles.

A cet effet il apparaît nécessaire de favorišer la réinstallation rapide d'une végétation naturelle diversifiée; elle seule permettra en effet l'intégration harmonieuse du site dans le paysage. Le biotope ainsi reconstitué entraînera aussi, de ce fait, le développement d'une faune associée diversifiée (avifaune, ichtyofaune, invertébrés...). Sa réutilisation à des fins touristiques et surtout halieutiques sera par conséquent facilitée et réalisable à moindres frais.

Le réaménagement le plus favorable à la restauration du milieu est donc le réaménagement naturel à des fins halieutiques. Il permet un compromis intéressant entre la nature et une exploitation raisonnable du milieu. D'autres activités ne pourront être superposées (voile, motonautisme), que si elles ne perturbent pas l'utilisation primordiale du milieu (bétonnage, destruction des herbiers, etc...).

(1) Institut Européen d'Écologie, 1 rue des Récollets, 57000 METZ.

(2) Laboratoire de Génétique Écologique, Unịversité des Sciences et Techniques de Lille, 59655 VILLENEUVE D'ASCO CÉDEX. 
Une conception adéquate de l'aménagement doit aborder les trois points suivants :

1) définir la taille et la forme du plan d'eau nouvellement créé en fonction de sa vocation future, de l'environnement naturel et potentiel du site;

2) définir le profil des berges:

3) bien choisir les espèces végétales à introduire et en particulier les essences ligneuses.

\section{TAIlle, forme des plans d'EAU, PROFONDEUR ET QUALITÉ DE L'EAU}

La taille doit être fonction de l'environnement immédiat du site :

- paysage fermé ou compartimenté (marais, bocage, semi-bocage) : petits plans d'eau. Ex. : vallée de la Somme.

- paysage ouvert (openfield): grands plans d'eau.

La configuration doit être irrégulière:

- Il faut éviter les angles géométriques, les berges régulières. II faut au contraire favoriser les configurations souples, les formes arrondies.

Il est intéressant tant pour la flore que pour la faune de créer des presqu'îles ou des îlots (figure 1).

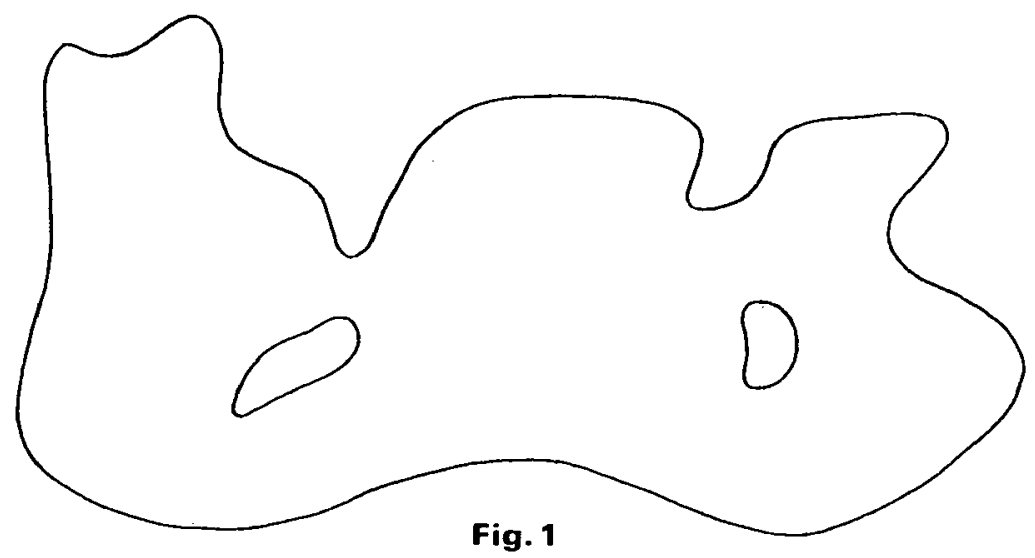

La profondeur du plan d'eau ne devrait pas excéder 3 mètres. La moyenne serait optimale à 2 mètres (les herbiers de Phanérogames se maintiennent bien à cette profondeur; au-delà, seules quelques Charophytes se développent). Dans ces conditions, l'évolution naturelle par comblement, qui s'effectue lentement, n'aura pas un impact sensible à moyen terme.

La qualité de l'eau est évidemment décisive dans le parti qu'on peut tirer d'un plan d'eau. A des fins halieutiques, les nappes dont les $\mathrm{pH}$ sont compris entre 6 et 8,5 sont les plus favorables (nappes alluviales, nappe de la craie); leurs eaux permettent l'installation d'une végétation phanérogamique diversifiée et structurée, elle-même nécessaire à l'installation des frayères. Les nappes de $\mathrm{pH}$ voisin de 5 (ex.: issues des sables landéniens) sont nettement moins favorables aux espèces régionales et au plancton.

\section{PROFIL DES BERGES}

Les berges ne doivent pas être abruptes : des pentes fortes peuvent rendre les abords dangereux en raison des éboulements. et d'autre part, elles rendent impossible la réalisation des différentes ceintures végétales naturelles potentielles.

La pente doit être au départ faible, inférieure à $10^{\circ}(7 / 1)$; un pallier joue le rôle de haut fond sur une largeur variable ( 2 à $5 \mathrm{~m}$ au minimum) de façon à favoriser l'installation des groupements d'hydrophytes des eaux peu profondes et des roselières. Une seconde pente plus forte, mais n'excédant pas $30^{\circ}(3 / 1)$, y fait suite (figure 2 ). La largeur de la zone garnie de végétation doit être fonction de la configuration et de la superficie du plan d'eau. Elle peut varier dans un ordre d'idées moyen entre 10 et $40 \%$ de la largeur du plan d'eau. 


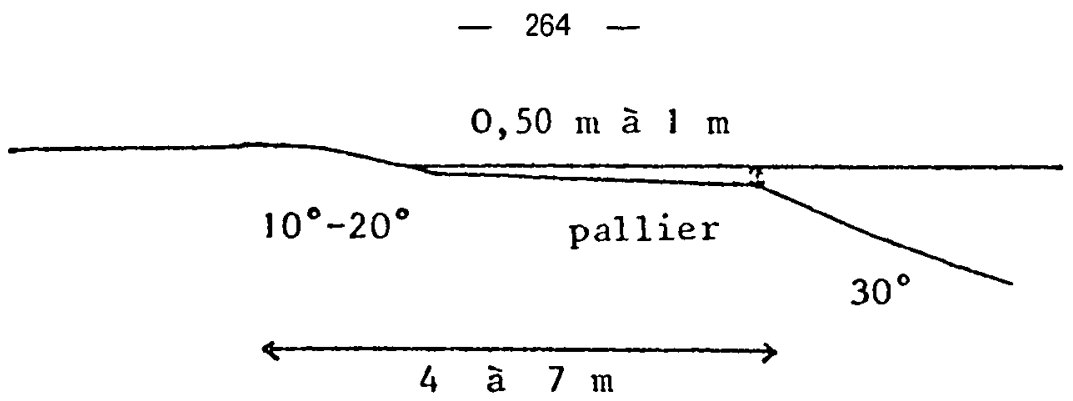

Fig. 2

En règle générale, il ne doit pas y avoir création de banquette périphérique en bourrelet saillant perturbateur dans le paysage alluvial (qui est fondamentalement plat et doit le rester). Ce bourrelet souvent haut de plusieurs mètres serait vite colonisé par les espèces nitrophiles anthropiques (Ortie, Cirse, Chardon); ainsi, les berges doivent rester à un niveau voisin de celui des prairies hygrophiles environnantes (contexte bocager) ou des marais adjacents (contexte marais). Cela permet dans la plupart des cas de retrouver la difficulté d'accessibilité antérieure du site (en particulier accès interdit aux automobiles) et c'est là l'une des bases d'une correcte cicatrisation du site (paysagère et biologique).

Dans le cas particulier d'une zone alluviale soumise à des crues saisonnières, il y a lieu de prévoir les submersions temporaires avec les conséquences qu'elles entraînent (échanges eau-poissons avec le milieu extérieur, apports de matériaux alluvionnaires, érosion locale des ouvrages sur berges). Si ces conséquences sont dommageables, il y a lieu de mettre le plan d'eau hors crue par des techniques appropriées (contre-canaux périphériques)

Les plans d'eau à vocation halieutique doivent être équipés de pontons en rondins; les aménagements à base de béton ou de matériaux métalliques sont à proscrire.

Les plans d'eau à vocation de promenade ou pédagogique sont à équiper de chemins éloignés du plan d'eau; ils ne doivent comporter que des "antennes" doubles ou simples permettant une bonne vision d'ensemble du site.

Des secteurs doivent demeurer inaccessibles aux promeneurs.

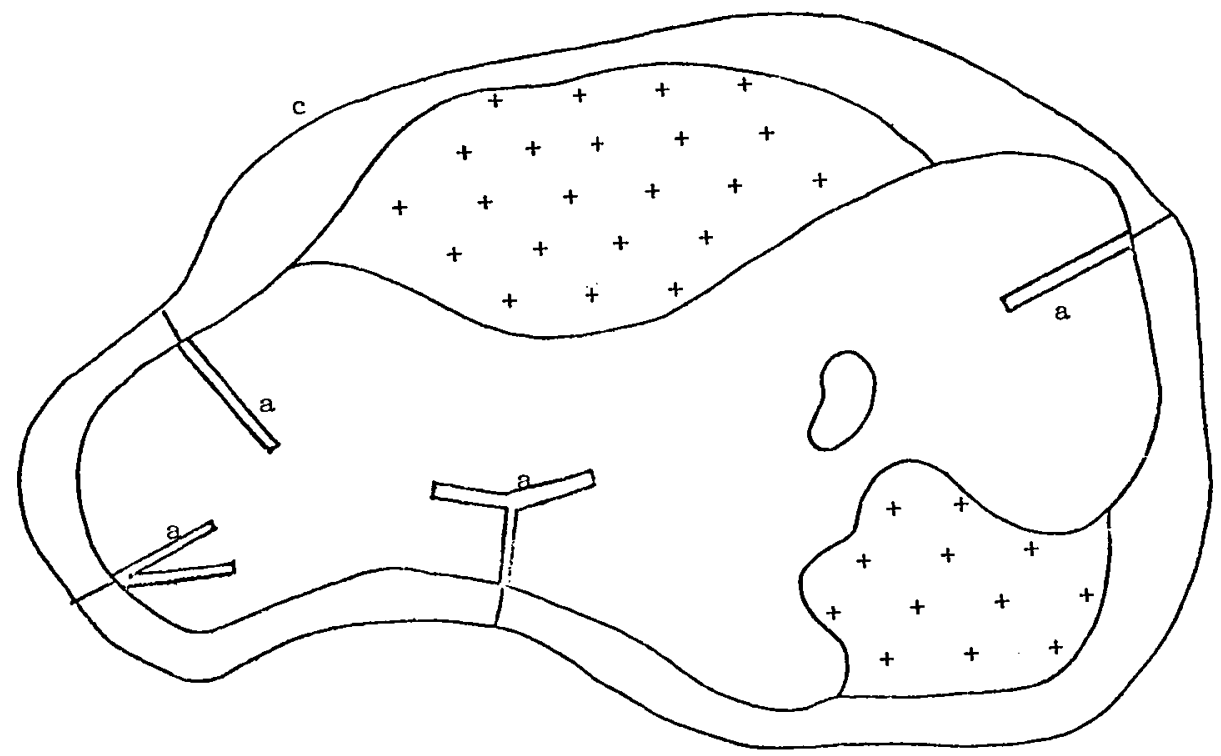

Fig. 3 : + : secteurs inaccessibles; a : antennes; $c$ : chemin de promenade et d'observation.

Les pavillons légers à but pédagogique devraient être réalisés en matériaux naturels dans un but d'intégration : bois (rondins), chaume (Roseau, Jonc des tonneliers). 


\section{ESPĖCES VÉGÉTALES A INTRODUIRE}

Exemple : vallée de la Somme, de l'Oise.

A - Les espèces aquatiques (hydrophytes) et subaquatiques (hélophytes) colonisent spontanément le plan d'eau si les berges sont peu pentées et si elles disposent de replats (hauts fonds).

Ces végétations hydrophytiques et hélophytiques assument plusieurs rôles essentiels:

- elles constituent des frayères indispensables à l'ichtyofaune ;

- elles réalisent un abri indéniable pour la faune des Invertébrés (Insectes et larves, Crustacés et larves, Mollusques...);

- elles produisent l'oxygène nécessaire aux animaux prélevant l'oxygène dissous pour leur respiration ;

- elles assurent pour une grande partie l'épuration biologique des eaux :

- elles déterminent l'installation d'une avifaune spécifique, sa diversité et sa richesse en espèces.

\section{Hydrophytes:}

Nénuphar blanc (Nymphaea alba)

Nénuphar jaune (Nuphar lutea)

Myriophylle en épis (Myriophy/lum spicatum)

Myriophylle verticillé (Myriophyllum verticillatum)

Elodée du Canada (Elodea canadensis)

Potamot luisant (Potamogeton lucens)

Potamot crépu (Potamogeton crispus)

Callitriche à fruits plats (Callitriche platycarpa)

Callitriche à angles obtus (Callitriche obtusangula)

Chara vulgaire (Chara vulgaris)

Renoncule velue (Ranunculus trichophyllus)

Lentillè d'eau en fer de lance (Lemna trisulca), à l'exception des autres espèces de lentilles

Renoncule peltée (Ranunculus peltatus)

Châtaigne d'eau (Trapa natans)

\section{Hélophytes:}

Les profondeurs mentionnées sont les conditions de la période printannière.

Jonc des tonneliers (Scirpus lacustris) - Profondeur : $2 \mathrm{~m}$

Massette à feuilles étroites (Typha angustifolia) - Profondeur : 0,60 m

Grande Glycérie (Glyceria maxima) - Profondeur : 0,50 m

Laîche des rives (Carex riparia) - Profondeur : 0,40 m

Massette à larges feuilles (Typha latifolia) - Profondeur: $0,30 \mathrm{~m}$

Roseau commun (Phragmites australis) - Profondeur : $0,30 \mathrm{~m}$

Laîche des marais (Carex acutiformis) - Profondeur : 0,20 m

Ces végétaux peuvent être aisément prélevés dans les étangs ou marais voisins, dans lesquels, par exemple, une autre carrière va s'ouvrir; le coût de l'opération ne représente que celui de la main d'cuvre et du transport.

\section{B - Les essences ligneuses à implanter:}

Ėles doivent essentiellement être locales ou régionales. Elles doivent être installées sous forme de groupements structurés, préfiguration du modèle naturel, de façon à accélérer la reconstitution du milieu originel.

1) au contact de l'eau et jusqu'à $2 \mathrm{~m}$ au-dessus du plan d'eau de printemps (zone des bois blancs, longuement inondable):

- eau stagnante: Saulaie à Saule cendré (Salix cinerea), Saule à oreillettes (Salix aurita), Saule à nervures multiples (Salix x multinervis), Bourdaine (Rhamnus frangu/a).

- eau en mouvement: Saulaie à Saule des vanniers (Salix viminalis) et Saule à 3 étamines (Salix triandra).

- dans les 2 cas : Saule blanc (Salix alba), Aulne glutineux (Alnus g/utinosa) et à un degré moindre Saule rouge (Salix rubens) sont à espacer de $2 \mathrm{~m}$ en $2 \mathrm{~m}$ en suivant les limites du plan d'eau ou à implanter en "bouquets" (Aulne) 
Insistons surtout sur le Saule blanc (Salix alba), magnifique espèce capable de " paysager" rapidement vu sa remarquable croissance et sa facilité de bouturage (fragments de $2 \mathrm{~m}$, gros de 5 à $10 \mathrm{~cm}$ ).

Les végétations hygrophiles arborescentes ont avec les végétations hélophytiques un rôle de protection des berges important.

2) en retrait de ces groupements à partir de $2 \mathrm{~m}$ à $3 \mathrm{~m}$ au-dessus du plan d'eau de printemps (zone des bois durs sur terrasses alluviales, périodiquement inondables):

- Aulnaie - Frênaie, Frênaie pure et Chênaie - Frênaie - Ormaie, avec Aulne, Frêne élevé (Fraxinus excelsior), Orme (UImus campestris) - ce dernier atteint actuellement par la Graphiose, maladie due à un champignon, ne doit pas être planté - Chêne pédonculé (Quercus robur). Peuplier grisard (Populus canescens), Erable champêtre (Acer campestre).

Les arbustes pouvant former des haies mêlées dans cette zone sont le Cornouiller sanguin (Cornus sanguinea), la Viorne obier (Viburnum opulus), le Prunellier épineux (Prunus spinosa), le Merisier à grappes (Prunus padus) - ce dernier a une floraison très esthétique -, le Saule cendré (Salix cinerea), I'Erable champêtre (Acer campestre), les Aubépines (Crataegus monogyna et $C$. oxyacantha), le Fusain d'Europe (Evonymus europaeus), le Noisetier (Corylus avellana), le Lierre (Hedera helix) - élément toujours vert formant une trame intéressante dans la haie -, le Houblon sauvage (Humulus lupulus). - Les espèces en caractères gras sont prioritaires -

Du point de vue des arbres, insistons sur le Chêne pédonculé, espèce arborescente de base dans nos paysages de plaine, qui devrait être l'espèce essentielle de paysagement depuis les milieux humides jusqu'aux milieux secs. Outre la qualité du paysage due aux Chênes isolés ou en "bouquets" de dimensions variables, c'est l'espèce-clef qui appelle immédiatement dans son environnement tout le cortège végétal et animal régional dont la réinstallation est d'ailleurs spontanée. Le Frêne doit lui être associé. La tradition séculaire du maintien du Chêne pédonculé dans les campagnes est la base de la très haute qualité des paysages de l'Angleterre du Sud, de l'Ouest français (Normandie surtout) et de l'Allemagne du Nord.

Finalement, Saule blanc ( 0 à $2 \mathrm{~m}$ au-dessus de l'eau) et Chêne pédonculé (au-delà de $2 \mathrm{~m}$ ) sont les deux éléments qui doivent former le fond des réinstallations paysagères sur les bords des carrières réaménagées.

Le coût de ces implantations est faible en raison de la facilité de se procurer ces essences locales.

Exemple: Saule blanc planté sous forme de boutures prélevées sur des Saules taillés en tétard, très nombreux dans cẹs vallées (jusque 100 boutures sur un même arbre).

Ces espèces locales sont nettement moins onéreuses que les essences exotiques.

Leur taux de reprise est voisin de $100 \%$, ce qui est loin d'être le cas des espèces étrangères; elles assurent par définition l'intégration du site dans le paysage qu'elles valorisent de surcroît, apportant par là un élément compensatoire fondamental conjurant partiellement la détérioration du site.

Le Peuplier américain (Populus x canadensis) est à rejeter : il dégrade fortement les sites à brève échéance (réduction de la flore accompagnatrice à 2 ou 3 espèces ainsi que de la faune). C'est une espèce à réserver aux plantations de production industrielle et non pas à "parsemer "en arbres isolés ou en plantations restreintes, en raison des effets alors très négatifs sur la qualité et l'harmonie des paysages.

Les Conifères (Epicéa, Chamaecyparis, Thuya) et les buissons exotiques toujours verts (Troène du Japon, Laurocerasus) sont à proscrire formellement. On peut voir, à l'ouest de Soissons, des réaménagements utilisant ces espèces et présentant un très mauvais aspect, en raison précisément de ces introductions, de la géométrie des contours et des clôtures trop voyantes.

Enfin, chaque cas de réaménagement est un problème particulier qui demande la prise en compte de réalités variées. Celles-ci s'opposent parfois au rétablissement total des valeurs naturelles. Mais ce réaménagement peut toujours intégrer un minimum de précautions paysagères et biologiques qui valorisent les différentes possibilités d'utilisations ultérieures. 


\section{C-Techniques d'implantations}

L'implantation artificielle de végétaux aquatiques est difficile à réaliser. Elle se fera en début de période végétative (avril à juin). Le fragment de l'association où figure l'espèce caractéristique sera introduit sous une cage de grillage plastifiée, de $1 \mathrm{~m} / 1 \mathrm{~m}$ et de $0,50 \mathrm{~m}$ de haut, de façon à ce que les hydrophytes ne remontent pas à la surface et développent leur système radiculaire. L'enclos protège aussi contre l'action des Anatidés et du Rat musqué, rongeur colonisant rapidement les milieux neufs ; il devra être enlevé au bout de 1 à 2 semaines.

Dans l'installation des végétaux, la nature du substrat et sa granulométrie jouent un rôle important. Si la taille des éléments est supérieure au centimètre, la réussite sera certainement meilleure en recouvrant le substrat de quelques centimètres de terre végétale, d'autant que le substrat neuf n'apporte que peu d'éléments nutritifs.

La plantation du Roseau (Phragmites australis) et des Laîches (Carex) s'effectuera en ballots; celle des Massettes (Typha angustifolia et $T$. latifolia), du Jonc des Tonneliers (Scirpus lacustris) et de la grande Glycérie (Glyceria maxima) par plantation de rhizomes et de rejets. La Glycérie peut être aussi semée sur des sols à condition que la surface ensemencée ne soit pas inondée pendant les six premiers mois qui suivent. Pour le Roseau commun on peut aussi enfoncer 3 à 4 boutures dans un trou de $0,30 \mathrm{~m}$ à $0,50 \mathrm{~m}$ de profondeur. Ces plantations se feront en automne ou en tout début du printemps.

Les Saules seront introduits soit individuellement sous forme de boutures, de verges, ou de perches, soit assemblés par tressage ou ligature par du fil de fer (fascines), soit encore par couche d'étendage comprenant des verges de Saules de 2 à 3 ans longues de $1,50 \mathrm{~m}$ à $2 \mathrm{~m}$, inclinées vers l'aval de la berge. Les implantations se feront de novembre à mars.

Les espèces de "bois dur" seront installées par la méthode classique de plantation de plants enracinés. En mélangeant différentes essences arbustives et arborescentes, on doit obtenir par des coupes appropriées une structure semblable au modèle naturel.

\section{CONCLUSION}

Etangs de pêche ou de chasse, plans d'eau nautiques, sites de plaisance périurbains : quelle que soit la destination qu'on voudra donner aux carrières en eau après exploitation, il apparaît fondamental de remodeler et surtout de végétaliser avec des normes biologiques et paysagères. Par delà l'aspect légal de l'aménagement compensatoire, il faut y voir la plus-value en matière d'exploitation ultérieure qu'apportera le rétablissement adéquat de la flore, de la faune et des paysages naturels. 